\title{
Search for natural susy
}

\section{Gabriella Gaudio*}

INFN-Pavia

E-mail: gabriella.gaudio@pv.infn.it

\section{Maurizio Pierini}

\section{CERN}

E-mail: maurizio.pieriniecern.ch

First searches at the LHC experiments have excluded the existence of supersymmetric particles in a large portion of the energy range reachable by the present machine conditions. The discovery of the Higgs bosons at relatively low mass imposes constraints on the SUSY particles masses which contribute to the Higgs mass corrections. The so called Natural SUSY models have been suggested to provide still viable solution for the hierarchy problem in the SUSY framework. These models have been adopted as guidance for the ATLAS and CMS searches. In the following, after briefly discussing the limits obtained by the experiments, we report on perspective of the analysis in this field.

VI Italian workshop on p-p physics at the LHC,

8-10 May 2013

O Acquario di Genova, Ponte Spinola, Area Porto Antico, Genova, Italy

\footnotetext{
${ }^{*}$ Speaker.
} 


\section{Results from ATLAS and CMS}

In the Supersymmetric model, the Higgs mass receives corrections from loops of particles such as gluinos, third generation squarks (stop and sbottom) and of the higgsinos [1]. Therefore, at least those particles in the SUSY spectrum should be lighter than the TeV scale for Supersymmetry (SUSY) to be the mechanism by which the Higgs vacuum is stabilized.

ATLAS and CMS experiments have addressed the search of those particles. considering both direct and gluino mediated production for third generation squarks, and ewkinos production. As a guidance for the analysis optimizations and interpretations, several simplified models have been used $[2,3,4]$. All the analysis are based, mainly on cut and count approach. The results have been interpreted in R-parity conservation scenarios and, since no excess with respect to the Standard Model expectation have been observed, limits on the mass and cross section production for sparticles have been derived.

Gluino mediated searches look for multijet (more than 6) events with large missing transverse energy. Different final states with or without leptons and/or b-jet are considered, depending on the decay mode of the stop/sbottom. Combination of the results for different searches excludes gluinos up to about $1300 \mathrm{GeV}$, for low $(<400 \mathrm{GeV})$ neutralino mass, in both stop and sbottom channels. Comparable limits have been obtained by both ATLAS and CMS. Limits obtained by the CMS collaboration on stop mediated gluino production are shown in figure 1 bottom.

Strategies for the direct stop production search vary as a function of the stop mass. In fact the cross section for stop pair production falls rapidly with increasing stop mass, being of order of $10^{-3} \times \sigma_{t \tilde{t}}$ for $m_{\tilde{t}} \sim 500 \mathrm{GeV}$. In the region at high mass the cross section is therefore small and the top pair production is the main irreducible background. In the low mass region, instead, the stop signature is mostly identical to the $t \bar{t}$ final state, therefore one needs powerful discriminating variables. Moreover mass difference among sparticles is an important parameter for the kinematic of the events, highly affecting the analysis sensitivity. Example of limits for the stop direct search obtained by the ATLAS collaboration in shown in figure 1 top. Stop with mass up to about 400 $\mathrm{GeV}$ is excluded for low neutralino mass.

Direct chargino-neutralino pair production have also been searched for. Depending on the mass hierarchy and the mixing matrix of the gauge eigenstates, also in this case different final states can be predicted. Many scenarios have been considered by the two experiments. Different analysis have obtained a limit of $300-600 \mathrm{GeV}$ for quite low $(<200 \mathrm{GeV})$ neutralino mass.

\section{Perspective of the Natural SUSY search}

Figure 1 shows two examples of the limits obtained by the searches for Natural SUSY with $8 \mathrm{TeV}$ data. Limits derived by both the experiments are already quite stringent in term of the naturalness of the supersymmetric models, though they are derived in simplifiled model scenarios and general implications are difficult to derive. In view of the first long shutdown and of the increase of the energy in Run II, the strategy for natural susy search is revised. 

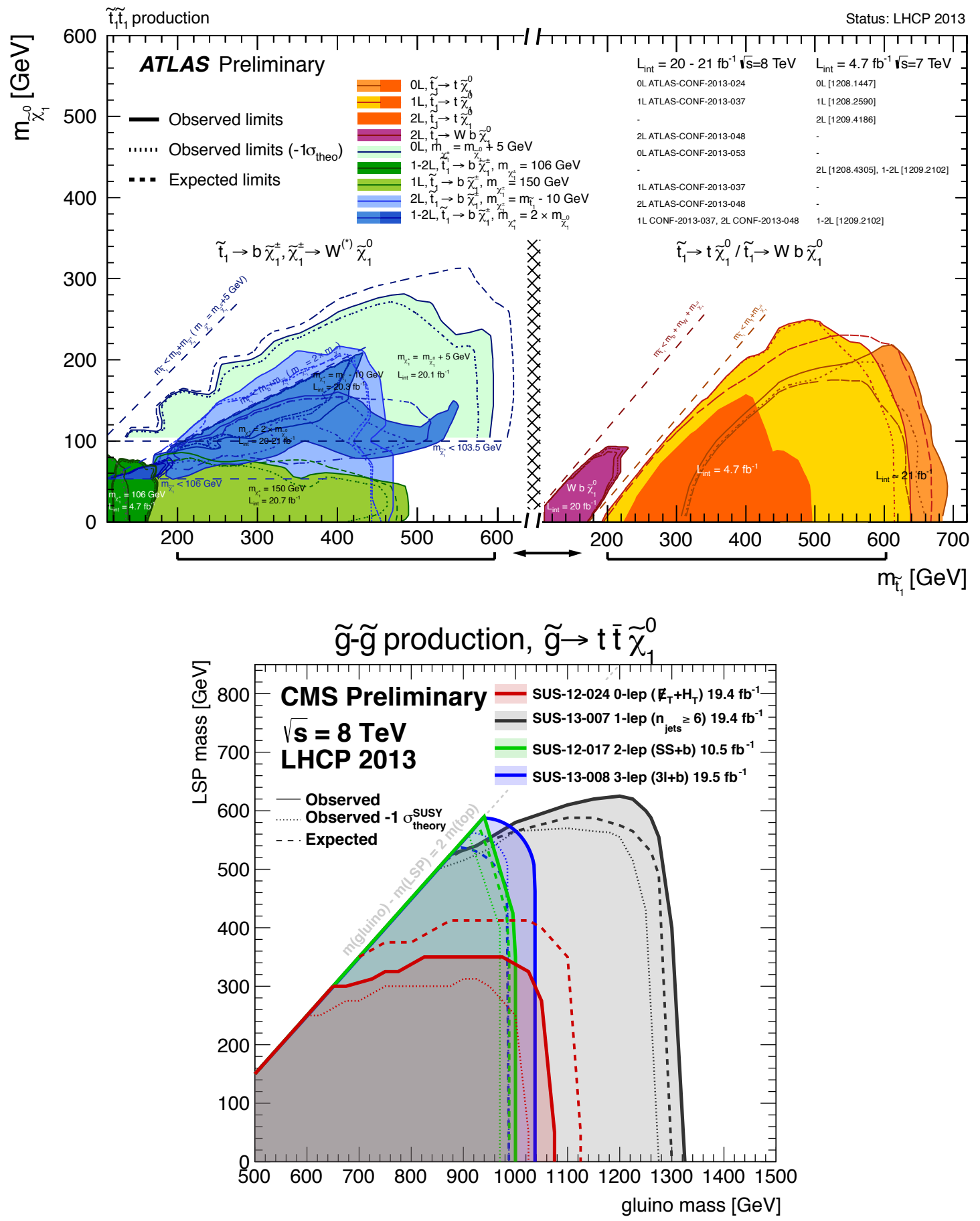

Figure 1: Limit on direct (top) [5] and gluino-mediated (bottom) [6] stop production as a function of the produced-sparticle mass and the LSP mass, obtained by ATLAS (left) and CMS (right). 


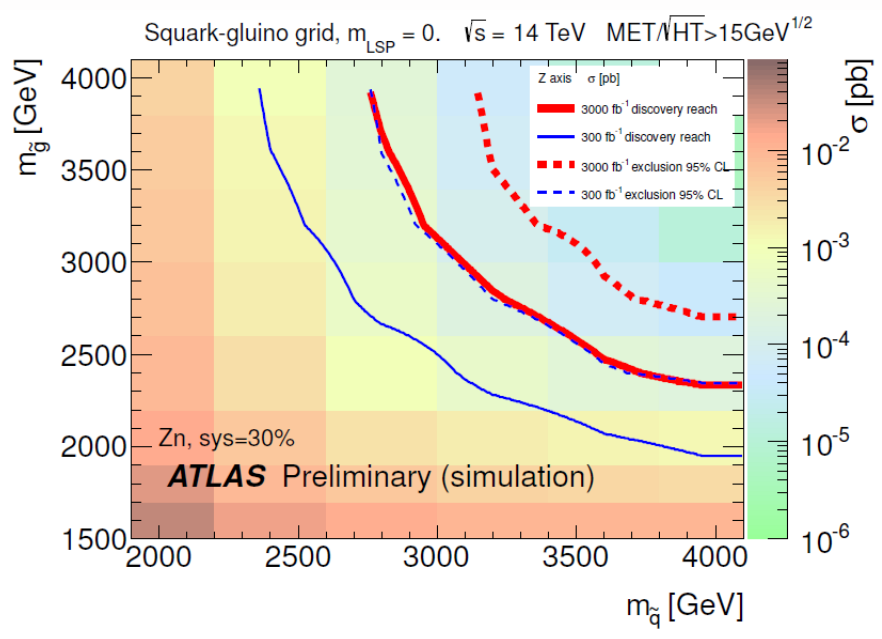

Figure 2: Extrapolated sensitivity of the ATLAS SUSY searches to squarks and gluinos at $14 \mathrm{TeV}$ for a dataset size of $300 \mathrm{fb}^{-1}$ and $3000 \mathrm{fb}^{-1}$.

\subsection{The missing corners}

The study of scenarios with compressed spectra, e.g. with stop-chargino-neutralino masses nearly degenerate, is difficult because of the very soft spectrum of lepton and jet foreseen in the decay chains. Search in the degenerate limits can only proceed through recoil of the sparticles produced against ISR, with a signature characterized by one or two jets plus large missing transverse energy, a signature common to the dark matter search. A challenge we should address in the coming $13 \mathrm{TeV}$ analysis is how to better trigger on this type of events.

As said, ATLAS and CMS mainly concentrated on R-parity conservation models. If this is not the case, the LSP would decay, giving rise to signatures with multijet, including a number of b-jet, but no missing transverse energy in the events. We could foresee and overlap with (multi)jet resonance searches, though for high multiplicity the combinatoric prevents seeing the resonance. Also in this case, triggering of those events could be a problem, especially for light-enough stop.

Another category of events in which a search for supersimmetry can be addressed is that with long lived particles (LLP), which is common to exotics searches. Low cross section and/or high mass could require more complex analyses (existing SUSY search + LLP tag)

\section{In preparation of RunII}

In 2015 LHC will resume the operations, delivering proton proton collisions at a center of mass energy of $13 \mathrm{TeV}$. ATLAS and CMS experiments are already preparing the strategy for the new energy frontier. Figure 2 shows the expected sensitivity on gluino and squark production assuming a dataset size of $300 \mathrm{fb}^{-1}$ and $3000 \mathrm{fb}^{-1}$ and no evidence for a SUSY signal.

\subsection{Exploit $8 \mathrm{TeV}$ data}

The analysis shown in the workshop have excluded large fraction of the space parameters in the low to mid range mass for gluinos, stop and sbottoms, charginos and neutralino. On the other hand, some uncovered regions still exist and their coverage should be addressed by further 
exclusive analyses on the $8 \mathrm{TeV}$ data.

Data collected in 2012 can also be used to validate both the Monte Carlo and the reconstruction. We should exploit the long shutdown to commission with data the tail of the distributions for the kinematic variables employed in the anlyses, such as missing transverse energy, total transverse energy, etc.. We also have a chance to improve our confidence on the Monte Carlo generation of ISR events, for example using a $t \bar{t}$ sample, which will be crucial to explore the compressed spectrum scenario, as described earlier. Standard Model studies with $8 \mathrm{TeV}$ data will also be of great importance in order to increase the level of control of the main backgrounds in our analysis.

\subsection{Preparing for $13 \mathrm{TeV}$ data}

Physics groups need to develop their analysis far in advance with respect of the moment in which the $13 \mathrm{TeV}$ data will be available. As a first step, inclusive analysis will be redone, also keeping in mind possible synergy with exotica groups.

In 2015 the trigger thresholds will be raised to cope with increasing rates. In order to retain a good sensitivity to signatures with low momentum particles, the design of new trigger strategy and the improvement of topological triggers are of great interest. Experiments need to be prepared to highstatistics analyses with low-energy specific signatures for compressed spectra, jet substructure, and multiplicity triggers.

\section{References}

[1] G. Villadoro "Supersimmetria dopo il run a $8 \mathrm{TeV}$ di LHC", these proceedings .

[2] J. Alwall, P. Schuster and N. Toro, Phys. Rev. D 79 (2009) 075020 [arXiv:0810.3921 [hep-ph]].

[3] J. Alwall, M. -P. Le, M. Lisanti and J. G. Wacker, Phys. Rev. D 79 (2009) 015005 [arXiv:0809.3264 [hep-ph]].

[4] D. Alves et al. [LHC New Physics Working Group Collaboration], J. Phys. G 39 (2012) 105005 [arXiv:1105.2838 [hep-ph]].

[5] ATLAS Physics results: https://twiki.cern.ch/twiki/pub/AtlasPublic/CombinedSummaryPlots/ATLAS_directstop_all_lhcp13.pdf

[6] CMS Physics results: https://twiki.cern.ch/twiki/pub/CMSPublic/SUSYSMSSummaryPlots8TeV/T1ttt_LHCP2013.pdf 